\section{Le régime occidental, l'obésité et la chirurgie bariatrique modulent l'anxiété, les habitudes alimentaires et la réponse cérébrale au goût sucré}

Yentl Gautier ${ }^{1}$, Damien Bergeat ${ }^{1,2}$, Yann Serrand ${ }^{1}$, Noémie Réthoré ${ }^{1}$, Mathilde Mahérault ${ }^{1}$, Charles-Henri Malbert ${ }^{3}$, Paul Meurice ${ }^{1}$, Nicolas Coquery ${ }^{1}$, Romain Moirand ${ }^{1,4}$, David Val-Laillet ${ }^{1}$
INRAE, Inserm, Univ Rennes,

CHU de Rennes, NuMeCan (Nutrition Métabolismes Cancer), 35590 St Gilles, France.

${ }^{2} \mathrm{CHU}$ Rennes, Service de chirurgie hépatobiliaire et digestive, Rennes, France.

${ }^{3}$ INRAE, US1395, AniScan, 35590 St Gilles, France.

${ }^{4} \mathrm{CHU}$ de Rennes, Service des maladies du foie et addictologie, 35033 Rennes, France.

david.val-laillet@inrae.fr
> La surconsommation d'aliments hautement palatables ${ }^{1}$, riches en gras et en sucre (régime occidental, en anglais Western diet), constitue un risque majeur de développement de l'obésité par la mise en place de dépendances alimentaires qui modifient la fréquence, la quantité et la structure des repas en faveur d'épisodes de pulsion alimentaire (craving) et de grignotages récurrents. En effet, la qualité et la quantité de nos apports nutritionnels entretiennent un rapport d'influence réciproque avec le contrôle central du comportement alimentaire, mais également de l'humeur (anxiété, dépression, etc.) et des facultés cognitives (apprentissage, mémoire, contrôle inhibiteur, etc.) grâce à la plasticité neuronale. Cette dernière est connue pour être particulièrement réactive lors des périodes critiques ou sensibles du développement des individus, telles que la période périnatale ou l'adolescence. Tous ces traits comportementaux sont modulés par des processus cérébraux dynamiques qui gèrent les dimensions cognitives, affectives, et hédoniques du comportement.

1 Se dit d'un aliment qui procure une sensation agréable lors de sa consommation.
Cette dynamique impliquerait en premier lieu un processus « d'excès de récompense » (reward surfeit theory) [1], qui a été observé chez des adolescents à risque de développer une obésité : la présentation d'images alimentaires à ces adolescents provoque une réponse accrue du striatum dorsal, sous-tendant une forte attente (anticipation) dans cette population. S'y ajouterait un mécanisme d'hypersensibilisation aux stimulus alimentaires (incentive sensitization theory) [2] qui favoriserait I'apparition et le maintien de l'hyperphagie. Enfin, il pourrait également exister un déficit de récompense (reward deficit theory) [3] responsable d'une hyposensibilité du circuit de la récompense en réponse aux stimulus alimentaires, en particulier lors de la consommation: cette hyposensibilité s'apparente aux mécanismes de dépendance aux drogues et contribuerait à l'escalade de la surconsommation pour compenser cette déficience.

La combinaison de ces trois théories a donné naissance au modèle de vulnérabilité dynamique de l'obésité, proposé par Burger et Stice (dynamic vulnerability model of obesity) [4]: ces auteurs suggèrent l'existence d'un basculement (shift) des processus hédoniques et cognitifs, permis par la plasticité neuronale et conditionné par l'alimentation.

À la lumière de ce modèle, nous avons émis l'hypothèse que l'exposition chronique à un régime occidental sans apparition d'une obésité serait suffisant pour induire une dépendance alimentaire pour ces aliments, et que, chez des individus obèses, les processus cérébraux en lien avec le contrôle du comportement alimentaire seraient modulés différemment par une intervention de chirurgie de l'obésité (bypass gastrique de type Roux-en-y) comparée à un régime restrictif.

Dans une étude réalisée chez des miniporcs Yucatan adolescents, nous avons reproduit le processus dynamique qui conduit, à partir de la surconsommation chronique d'aliments gras et sucrés (Western diet), d'abord sans prise de poids (statut normopondéral, étude $\mathrm{l}^{2}$ ), au développement de l'obésité consécutif à un accès ad libitum à ce régime alimentaire (étude 2). Le protocole s'achève par une perte de poids (weight loss) induite soit par un régime

\footnotetext{
2 Pour l'étude 1 , nous avons comparé un groupe de miniporcs exposés à un régime alimentaire de type Western diet à un autre groupe sous régime standard équilibré (standard diet).
} 


\begin{tabular}{|c|c|}
\hline $\begin{array}{c}\text { Pourcentage d'excès de poids } \\
\text { par rapport à la norme santé (en \%) }\end{array}$ & Catégorie \\
\hline $0-20 \%$ & Surpoids \\
\hline $20-40 \%$ & Obésité modérée \\
$40-60 \%$ & Obésité sévère \\
$>60 \%$ & Obésité morbide \\
\hline
\end{tabular}

Tableau I. Définition du statut pondéral des animaux utilisés dans l'étude d'après l'échelle humaine IMC (indice de masse corporelle). Le pourcentage d'excès de poids par rapport à la courbe de croissance normale théorique a été utilisé pour caractériser le degré d'obésité des animaux. Les pourcentages ont été calculés d'après le poids normal maximum pour une taille (hauteur) donnée.

alimentaire restrictif après une intervention chirurgicale factice (groupe sham-operated), soit par la création chirurgicale d'un by-pass gastrique de type Roux-en- $y^{3}$. Ces deux interventions ont été associées à une restriction calorique et à un retour à une alimentation saine (standard diet), comparable à celle qui est appliquée aux patients ayant subi une telle intervention chirurgicale (étude 3). L'évolution pondérale des miniporcs est présentée dans la Figure IA. Une échelle fondée sur le pourcentage d'excès de poids par rapport à une fourchette normale a été proposée pour caractériser le statut pondéral des animaux (Tableau 1). Pour chacune des trois études, nous avons réalisé : un test de comportement alimentaire (test de choix ou préférence alimentaire $)^{4}$, qui nous a permis de caractériser les comportements alimentaires des animaux en termes de microstructure du repas, de préférence et de quantité d'aliments ingérée ; un

3 Les animaux ayant suivi un régime restrictif seul ont subi une chirurgie factice (sham-operated), c'està-dire une laparotomie sous anesthésie générale semblable à celle des animaux ayant eu un by-pass gastrique.

${ }^{4}$ Ce test de choix alimentaire, d'une durée de 10 minutes, consistait à présenter aux animaux deux aliments de texture différente de celle de leur aliment habituel (bouillie au lieu de granulés), I'un contenant $20 \%$ de saccharose, l'autre $2 \%$. Les quantités consommées et différents comportements étaient enregistrés. test cognitif («planche à trous », hole board en anglais $)^{5}$, qui permet d'évaluer le statut affectif des individus en plus des capacités d'apprentissage et de mémoire; et une imagerie cérébrale par tomographie par émission de positrons (TEP) pour évaluer la réponse métabolique des différentes aires cérébrales à une stimulation gustative sucrée (solution de saccharose sur la langue).

Le test cognitif a permis de mettre en évidence la dynamique du statut affectif en fonction des fluctuations pondérales des animaux, avec l'apparition d'un comportement de type anxio-dépressif chez les animaux devenus obèses. Cette dégradation émotionnelle s'est traduite par une perte d'intérêt des animaux obèses pour le test: les individus obèses ont passé plus de temps à longer les parois du parc et moins de temps à explorer le dispositif de test que les animaux au statut normopondéral (Figure IB). De plus, les miniporcs obèses ont aussi vocalisé davantage que lorsqu'ils avaient encore un poids

\footnotetext{
${ }^{5}$ Le test du hole board utilise une arène carrée dans laquelle sont disposées 16 auges recouvertes d'un ballon que l'animal doit soulever pour atteindre une récompense alimentaire très appétissante (M\&M's). Seules 4 auges sur 16 (toujours les mêmes pour un animal donné) contiennent une récompense accessible. Les animaux sont testés à de nombreuses reprises durant trois semaines, ce qui permet d'évaluer leur motivation pour la récompense ainsi que leurs capacités d'apprentissage et de mémorisation spatiale.
}

normal, ce qui traduit une recherche accrue de contacts sociaux, typique des animaux anxieux en situation d'isolement social (ce qui est le cas lors du test qu'ils effectuent individuellement). Ces comportements anxio-dépressifs se sont normalisés après que les animaux ont perdu du poids, de la même façon à la suite des deux types d'interventions (bypass gastrique ou régime alimentaire restrictif).

Nous avons par ailleurs constaté qu'une exposition chronique à un régime alimentaire du type Western diet, sans prise de poids, altérait significativement la mémoire de travail (forme de mémoire à très court terme) de ces animaux par rapport à ceux exposés à un régime sain du type standard diet. Les scores de mémoire ont en fait augmenté lorsque les animaux sont devenus obèses, mais l'interprétation de ce résultat surprenant doit prendre en compte la perte de motivation des animaux pour le test. En effet, le score de mémoire dépend directement du nombre d'erreurs commises pendant l'exploration du dispositif; or les animaux obèses ont beaucoup moins exploré le dispositif de test (au profit de l'exploration des parois, indiquant leur volonté de sortir du parc), et ont par conséquent commis moins d'erreurs. Cependant, nous avons constaté qu'à la suite de la perte de poids, et avec un niveau de motivation (donc d'exploration) rétabli, les animaux ont obtenu des scores de mémoire comparables à ceux qu'ils avaient obtenus lorsqu'ils étaient obèses, c'est-à-dire des scores élevés, avec une tendance à être supérieurs à ceux obtenus lors de l'étude l (mais la différence n'était pas statistiquement significative : $0,05<P<0,1$ ), dans laquelle l'exposition chronique au régime Western diet avait altéré leur mémoire de travail (Figure 1C).

Dans le test de préférence alimentaire (test de choix), les animaux recevaient une ration alimentaire complète avant le test : dans l'étude 1 , cette ration était donc bien moindre que dans l'étude 2 puisque, dans cette dernière, les ani- 

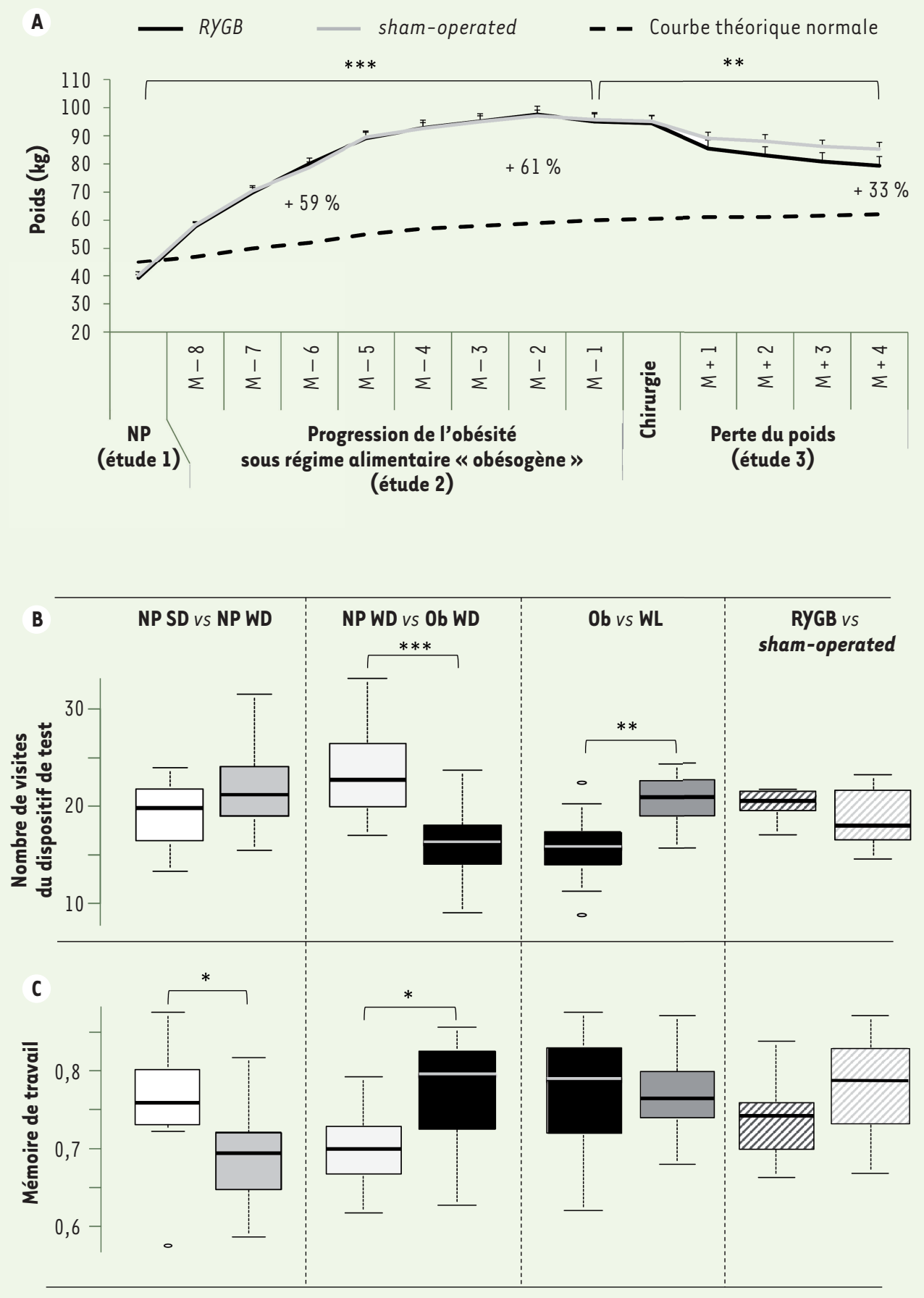

Figure 1. A. Évolution du poids vif ( $\mathrm{kg}$ ) des miniporcs ayant subi un by-pass gastrique de type Roux-en-Y (RYGB) ou un régime alimentaire restrictif (sham-operated) comparée à la courbe de croissance normale théorique en fonction du temps (en mois avant [《M-»] et après [《M+»] la chirurgie). Les pourcentages indiquent les taux d'excès de poids 6 mois et 2 mois avant la chirurgie $(M-6, M-2)$ et 4 mois après la chirurgie $(M+4)$. B. Nombre de visites du dispositif «planche à trous » (hole board) en fonction du régime alimentaire, régime standard (SD) ou du type Western diet (WD), du statut pondéral, normopondéral (NP), obèse (Ob), ou après perte de poids (WL, weight loss), et du type d'intervention, bypass et restriction calorique sous régime standard (RYGB), ou chirurgie factice et restriction calorique sous régime standard (sham-operated). Le groupe WL correspond au regroupement des animaux RYGB et sham-operated). C. Scores de mémoires de travail en fonction du régime alimentaire (SD ou WD), du statut pondéral (NP ou Ob) ou après perte de poids (WL), et du type d'intervention (RyGB ou sham-operated). Le groupe WL rassemble les individus RyGB et sham-operated. *:P<0,05; **:P<0,01; ***:P<0,001. 


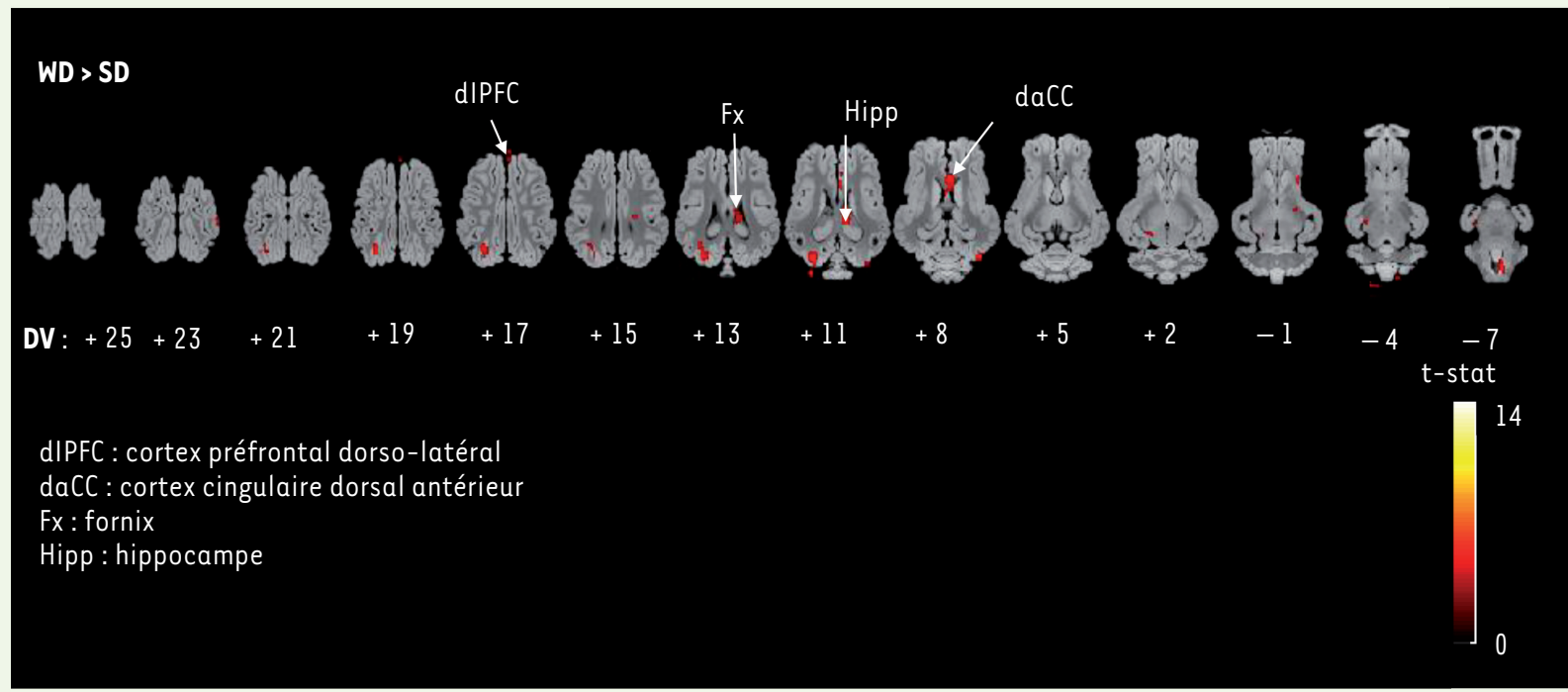

B

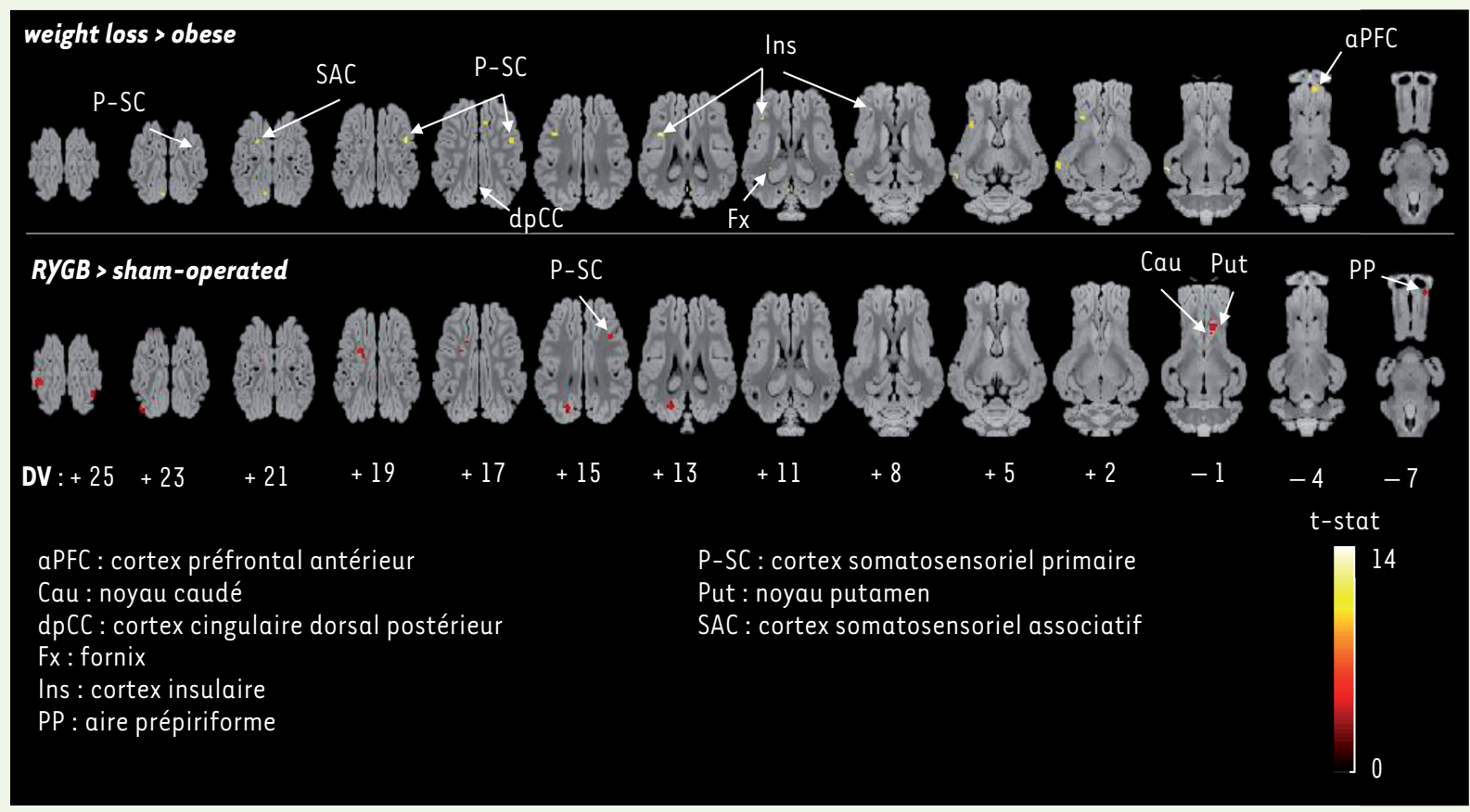

Figure 2. A. Cartes d'activation sur coupes horizontales du cerveau, obtenues par imagerie TEP (tomodensitométrie par émission de positrons) en réponse à une stimulation gustative sucrée orale, illustrant l'existence d'un métabolisme glucidique cérébral supérieur chez les animaux de poids normal sous régime alimentaire du type Western diet (WD) par rapport à des animaux témoins sous régime standard (SD) $(P<0,001)$. Ces différences s'observent notamment dans le cortex préfrontal dorso-latéral, le cortex cingulaire dorsal antérieur, l'hippocampe et le fornix. B. Cartes d'activation sur coupes horizontales du cerveau, obtenues par imagerie TEP en réponse à une stimulation gustative sucrée orale, illustrant l'augmentation du métabolisme glucidique cérébral, d'une part chez les animaux après la perte de poids ayant suivi l'intervention chirurgicale (animaux RYGB et sham-operated regroupés) par rapport à leur situation à l'état obèse avant l'intervention ( «eight loss > obese »; $P<0,05)$, et, d'autre part, chez les animaux RYGB comparés aux animaux sham-operated ( $R Y G B>$ sham-operated $» P\langle 0,001$ ). Ces différences s'observent notamment au niveau du cortex préfrontal antérieur, des noyaux caudé et putamen, du cortex cingulaire dorsal postérieur, du cortex insulaire, de l'aire prépiriforme, du fornix, ainsi que du cortex somatosensoriel primaire et associatif. La position dorso-ventrale (DV) des coupes du cerveau est indiquée en mm par rapport à la commissure postérieure. L'échelle de couleurs ( $t$-stat) représente la valeur $t$ du test statistique de Student pour la comparaison entre voxels, la couleur jaune correspondant aux différences les plus significatives (valeurs de t les plus élevées). 
maux avaient un accès libre à l'alimentation. Comme attendu, les quantités consommées par les animaux obèses (étude 2) lors du test de choix ont été inférieures à celles mesurées lors de ce test lorsque les animaux étaient à l'état normopondéral (étude 1) puisque les niveaux de rassasiement avant le test étaient différents entre les deux études. En termes de comportement alimentaire, il faut cependant noter que les individus obèses ont consommé quand même des aliments, en dépit du rassasiement, et ont augmenté la fréquence des bouchées. Ainsi, ces animaux n'ont pas éprouvé de lassitude vis-à-vis de l'aliment proposé lors du test, alors qu'il était identique à celui de leur ration quotidienne, ce qui pourrait indiquer une addiction au sucre présent dans l'aliment. De plus, ils ont compensé l'impossibilité d'ingérer une grande quantité de l'aliment en augmentant le nombre de prises (bouchées) alimentaires, leur permettant ainsi de « grignoter » pendant toute la durée du test. Ce comportement de grignotage et de poursuite de la prise alimentaire en dépit du rassasiement est également observé chez l'être humain en situation de dépendance alimentaire ou d'obésité liée à des troubles du comportement alimentaire [5]. Après la perte de poids, en situation de restriction calorique, les animaux avec le by-pass gastrique ainsi que les animaux témoins (shamoperated) ont augmenté leur prise alimentaire. Bien que les premiers aient eu tendance à consommer moins que les seconds, dont l'estomac est intact, la différence reste statistiquement non significative $(0,05<P<0,1)$ et révèle une grande variabilité dans la réponse des animaux au by-pass gastrique, certains ingérant des quantités comparables à celles ingérées par les animaux témoins.

Bien qu'aucune différence de comportement alimentaire n'ait été observée entre les animaux de poids normal au régime alimentaire standard et ceux de poids normal au régime de type Wes- tern diet lors du test de choix alimentaire, l'imagerie TEP du cerveau a révélé, chez ces derniers, une augmentation du métabolisme cérébral (donc de l'activité neuronale) dans le cortex préfrontal dorso-latéral, le cortex cingulaire dorsal antérieur, et l'hippocampe, lors de la gustation de saccharose. Cette réponse cérébrale au goût sucré est très similaire à ce qui est observé en cas de dépendance chez l'Homme et dans plusieurs modèles animaux $[6,7]$. II semble donc que l'exposition chronique à un régime de type Western diet entraîne un profil d'activation cérébrale de type «addiction»lors de la perception gustative du sucre, mais sans conséquence comportementale observable en situation alimentaire contrôlée (rationnée). Ce profil d'activité cérébrale pourrait être à l'origine de l'apparition d'un comportement ressemblant à une dépendance, que nous avons observé dans le test de préférence alimentaire chez les mini-porcs devenus obèses par la suite (Figure 2A). La perte de poids a permis d'augmenter l'activité cérébrale de l'insula (notamment dans l'aire gustative), et du réseau cortico-hippocampique (fornix, cortex préfrontal) impliqué dans les réponses inhibitrices. Comparé au régime restrictif seul, le by-pass gastrique a augmenté le métabolisme du noyau caudé et du putamen. Une altération de la réponse du noyau caudé ayant été identifiée comme une cause des épisodes de pulsion alimentaire [8], une activité accrue de cette structure devrait permettre à l'individu d'inhiber plus efficacement sa prise alimentaire (Figure 2B).

Cette étude chez le miniporc Yucatan a permis de distinguer les effets d'une qualité d'alimentation dégradée sans prise de poids, de ceux de l'obésité. Par ailleurs, elle a permis de mettre en évidence les intérêts du by-pass gastrique par rapport à une restriction calorique simple, en soulignant cependant la forte variabilité des réponses chez les animaux ayant subi cette intervention chirurgicale. Enfin, elle confirme la pertinence de l'utilisation de ce modèle animal pour étudier certains mécanismes des troubles alimentaires et de l'obésité dans l'espèce humaine. La suite de l'étude dans ce modèle consistera à analyser les variations des statuts hormonal et bactériologique, et à étudier les adaptations intestinales. $\diamond$ Western diet, obesity and bariatric surgery modulate anxiety, eating habits and the brain responses to sweet taste

\section{LIENS D’INTÉRÊT}

Les auteurs déclarent n'avoir aucun lien d'intérêt concernant les données publiées dans cet article.

\section{RÉFÉRENCES}

1. Stice $\varepsilon$, Yokum S, Burger KS, et al. Youth at risk for obesity show greater activation of striatal and somatosensory regions to food. J Neurosci 2011 ; 31 : 4360-6.

2. Robinson $T \varepsilon$, Berridge $K C$. The incentive sensitization theory of addiction: some current issues. Philos Trans R Soc B Biol Sci 2008 ; 363 : 3137-46.

3. Wang GJ, Volkow ND, Fowler JS. The role of dopamine in motivation for food in humans: implications for obesity. Expert Opin Ther Targets $2002 ; 6: 601-9$.

4. Burger KS, Stice $\varepsilon$. Neural responsivity during soft drink intake, anticipation and advertisement exposure in habitually consumming youth : Neural response to soft drinks. Obesity $2014 ; 22: 441-50$.

5. Spitzer L, Rodin J. Human eating behavior: a critical review of studies in normal weight and overweight individuals. Appetite 1981; 2 : 293-329.

6. Davids S, Lauffer $\mathrm{H}$, Thoms K, et al. Increased dorsolateral prefrontal cortex activation in obese children during observation of food stimuli. Int J Obes 2010 ; $34: 94-104$

7. Vorel SR, Liu X, Hayes RJ, et al. Relapse to cocaineseeking after hippocampal theta burst stimulation. Science 2001 ; 292 : 1175-8.

8. Babbs RK, Sun X, Felsted J, et al. Decreased caudate response to milkshake is associated with higher body mass index and greater impulsivity. Physiol Behav 2013; 121: 103-11.
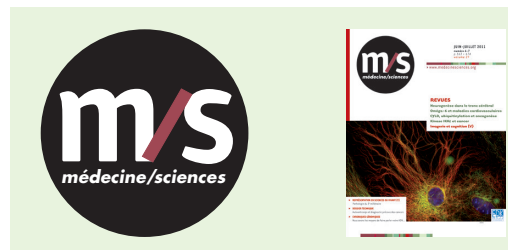

Abonnez-vous

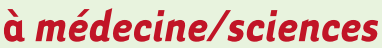

Bulletin d'abonnement page 222 dans ce numéro de $\mathrm{m} / \mathrm{s}$ 